\title{
Formación integral, inclusión educativa y responsabilidad social
}

No estoy por debajo de lo normal, ni valgo menos, ni soy menos capaz, sólo funciono de manera diferente.

Anónimo

\section{Resumen}

$\mathrm{E}$ s relevante para la educación hablar de inclusión y poder ser coherente en todo lo que involucran estas dos palabras en la sociedad. Inclusión es dar la bienvenida a cada ser humano al observarlo, conocerlo y darle la mano a través del acompañamiento en la etapa de desarrollo en que se encuentre; educación es un proceso a lo largo de toda la vida en el cual se forma y se potencia al ser humano en conocimientos, actitudes y habilidades, a través de la motivación o el gusto por aprender y al desaprender para estudiar por medio de la innovación o su propia capacidad. En este artículo se hace mención a una experiencia de cuatro años en los que, con objetividad, se comparten logros al llevar un modelo educativo por competencias en el sistema de preparatoria abierta en un Centro de Atención para Estudiantes con Discapacidad (CAED), ubicado en el сBтis No. 168 de la ciudad de Aguascalientes.

Palabras clave: formación integral, inclusión educativa, responsabilidad social.

\section{Introducción}

El sistema de preparatoria abierta en el Centro de Atención para Estudiantes con Discapacidad (CAED), ubicado en el Свтіs No. 168, es un proyecto que surge con la intención de brindar un espacio a jóvenes y/o personas que terminan la secundaria, quienes presentan alguna discapacidad visual, motriz, intelectual, auditiva y/o psicosocial, algunos de ellos con un promedio inferior a ocho o con el antecedente de haber recibido apoyo especial en su educación básica. La atención se ofrece de acuerdo a las necesidades específicas de la discapacidad presentada, por lo que es necesario acondicionar el espacio para su movilidad y brindar asesoría en grupos pequeños; además, hacer adaptaciones con base en el modelo curricular en que se imparte el bachillerato no escolarizado, conocido como preparatoria abierta, constituido por 22 módulos de las cuatro áreas disciplinares que a continuación se mencionan: comunicación, ciencias sociales y humanidades, ciencias experimentales y matemáticas.

$\mathrm{Al}$ tener una visión clara de la importancia de contribuir en la socioformación de los estudiantes, con la cual se "busca responder a los retos para formar a la sociedad del conocimiento, focaliza su atención en las actuaciones integrales de las personas ante problemas del contexto por medio de proyectos, desde el marco del proyecto ético de vida con procesos transversales" (Tobón, 2013, en Carrasco, 2016). El CAED se organiza con un programa anual en el que se integran actividades académicas y extracurriculares, como Crearte, Actívate y Desarrollo socioemocional, contribuyendo así a su formación integral, además de potencializar todas las áreas de desarrollo de las personas con discapacidad en este espacio.

\section{Desarrollo}

La inclusión educativa es un derecho para integrar a todo ser humano en la diversidad y observar a la humanidad con capacidades y talentos de manera individual, los cuales necesitan atención y adaptaciones en su formación para seguir favoreciendo su integridad. Es significativo comentar que, al recibir a las personas con discapacidad en la primera entrevista que se les hace de manera per- 
sonalizada, se observan sus miedos al maltrato, sus inseguridades, sentimientos de baja autoestima, las grandes etiquetas con las que se han ido minimizando al compararse con estándares que imponen lo "normal" a lo largo de su camino educativo, además de sentirse poco capaces por todas las historias que "vienen cargando". Al compartirles que, si ellos quieren estudiar, consideren éste su espacio, que son valiosos, podrán continuar sus estudios y encontrarán asesores y compañeros que los respetarán por lo que ellos son: personas capaces de acuerdo a sus necesidades y situación real. Se les explica cómo se trabaja el programa del CAED, los ambientes de aprendizaje que se crean por medio de un esquema de asesorías, en el que día a día se contribuye a la calidad educativa en todos los ámbitos que prevalecen en el desarrollo integral. Su expresión corporal cambia como arte de magia cuando son acompañados por sus padres de familia, sus ojos se llenan de lágrimas con una mano extendida de gratitud y maravillados al encontrar un aula que hace la diferencia y es inclusiva, con la esperanza de un mundo mejor. Es por ello que nace el lema que los caracteriza " Saber que se puede!".

Para Tobón (2013: 32), "la trasmisión de conocimientos es el objetivo de cualquier proceso educativo, pero no el único. La enseñanza de valores y actitudes es también fundamental". Por lo anterior, los asesores tienen un perfil del área de especialización que se requiere, de acuerdo a la preparatoria abierta; además, reciben una ca-

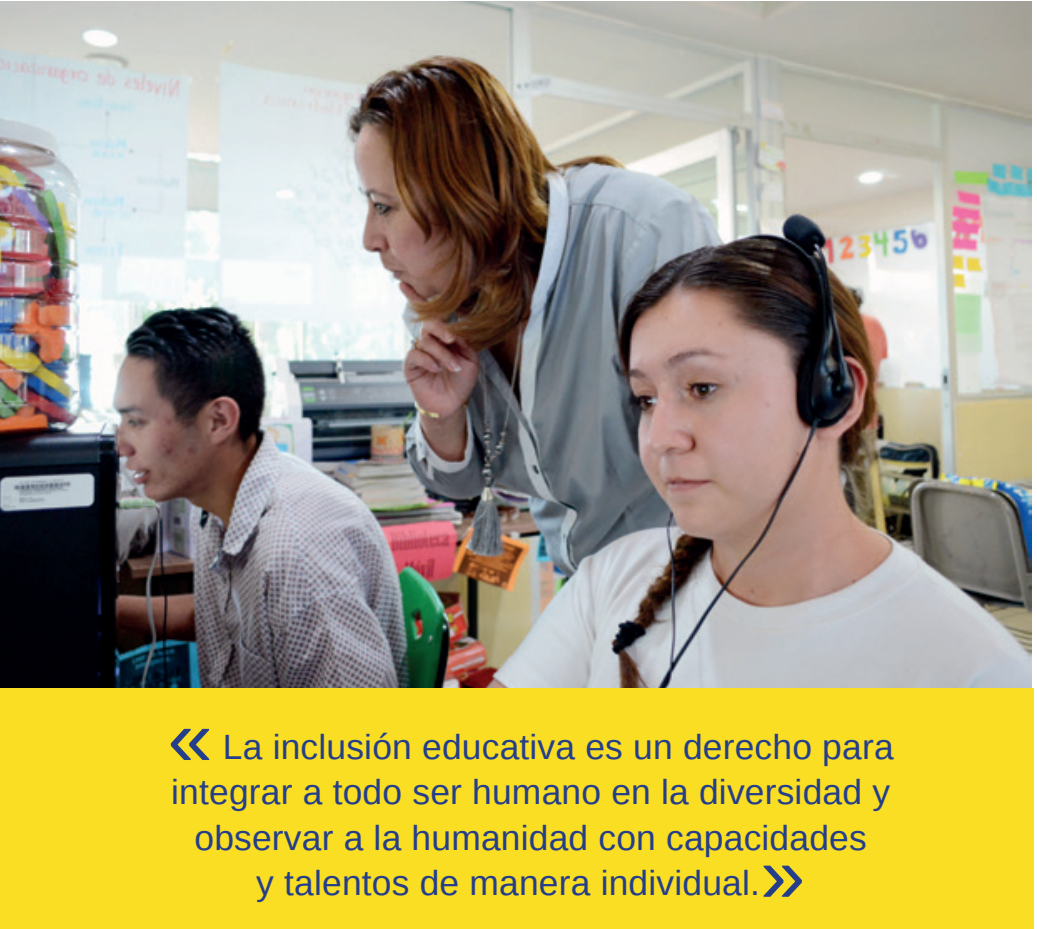

pacitación para contribuir en las asesorías de los estudiantes con discapacidad, adaptar sus planeaciones, estrategias de asesoría, evaluación y, principalmente, al momento de invitarlos al proyecto, se observa su sensibilidad y el gran trato humano, además de las expectativas al visualizar el lema del CAED, las oportunidades, las posibilidades para lograr su aprendizaje y desarrollo humano en la etapa educativa en la que se encuentran cada uno de los jóvenes, al seguir promoviendo sus talentos, además de desarrollar sus habilidades socioemocionales por medio del respeto y paciencia a su propio ritmo y cubrir sus necesidades de socialización.

En ocasiones, el docente se convierte en asesor académico y personal, por las necesidades propias de esta edad $\mathrm{y}$ al hecho de ir desenvolviéndose en un ambiente de crecimiento y confianza, se motiva también al logro de cada una de sus necesidades, además de elevar su autoestima y proceso de autonomía, que los lleva a tomar decisiones en su vida de manera trascendente. Lo anterior de acuerdo con Ceñal, Pérez y Ortega (2016: 16), quienes afirman:

El hecho de que se trate a cada aprendiz en términos de "persona apta para", con habilidades, capacidades, aptitudes y valores, modifica la clasificación superficial de "rendimiento bueno": todos se diferencian por sus contextos personales, por sus distintas características psicológicas, intelectuales, físicas y sociofamiliares. El aprendiz requiere de apoyo/ayuda psicoeducativa que va desde limitado e intermitente, flexible e individualizado, hasta general, permanente y continuo.

Por medio de las diferentes interacciones que se tienen día a día, se van demostrando mejores actitudes y creando vínculos intrapersonales e interpersonales, al interiorizar consigo mismos y darse cuenta de las necesidades y deseos de proyectarse por medio de las metas que se tienen de manera personal, además de sentirse afirmados por compartirlas y afianzar sus necesidades al ir socializando más fácilmente a su propio ritmo, así como a la aceptación de su decisión en el momento oportuno para llevar a cabo este proceso de aprender a convivir con diferentes personas en el medio que los rodea, con el propósito de ayudar en su crecimiento personal y desarrollo de sus habilidades sociales a través de la colaboración y el logro de objetivos en común. Es un proceso formativo donde intervienen los principales actores de la educación: docentes-asesores, alumnos-estudiantes y padres de familia-tutores.

En este sentido, los actores de la educación antes referidos asumen una gran responsabilidad social al estar 
frente a un estudiante, la cual se aprecia como una cualidad, un valor del ser humano, una característica positiva de las personas que son capaces de comprometerse y actuar de forma correcta y, al interactuar con más de una persona, se da el proceso de socialización del cual algunas de las personas con discapacidad están ávidas por vivenciar. Por ello, el docente es un ejemplo en la forma de mediar el aprendizaje y la interacción cotidiana con los estudiantes y los otros actores educativos, por medio de ambientes presenciales y, en ocasión, virtuales, contribuyendo al desarrollo integral de los estudiantes al incorporar estrategias que promuevan su desarrollo físico, intelectual, afectivo y social. Es importante percibir la sensibilidad en su vocación por el gran compromiso que tienen en el proceso educativo y el desarrollo del proyecto de vida de cada uno de los estudiantes.

Al mencionar parte de las experiencias del programa anual en el CAED, un asesor muestra la congruencia con este gran valor al desarrollar habilidades de empatía en el aprendizaje, planear de acuerdo a las necesidades que tiene en su grupo, desarrollar estrategias de enseñanza que susciten el gusto por el aprendizaje, elaborar ficheros autodidactas para promover la autonomía, crear evaluaciones adaptadas para ir monitoreando el proceso de aprendizaje, realizar material didáctico adaptado para que los estudiantes comprendan las lecciones de los módulos y sean más significativas e innovadoras, y sobre todo, encuentren la aplicabilidad en su vida diaria, como el desarrollo de hábitos, vivencia de valores, desarrollo de habilidades del pensamiento, resolución de problemas de acuerdo a su nivel metacognitivo, cuidado del medio ambiente, expresión artística en cualquier disciplina, así como conocerse a sí mismos por medio de aspectos socioemocionales, e interactuar en el medio ambiente a través de la asistencia a espacios culturales, deportivos, recreativos, instituciones de educación, etcétera.

Cabe resaltar que por la etapa de adolescencia en la que prevalece el mayor número de los estudiantes, se tiene proyectado establecer rutinas para que experimenten su autorregulación personal. Desde que llegan al CAED, registran su asistencia, disfrutan su asesoría y realizan una autoevaluación para valorar su proceso formativo, además de hacerlos reflexionar en conciencia de sus actos por medio de una disciplina efectiva (en la que se acompaña su proceso de concientización cuando se suscita alguna situación de conflicto en las relaciones interpersonales); igualmente, es importante compartir el hecho de que también los asesores realicen como parte de su reflexión y autorregulación el portafolio docente y la bitácora como instrumentos para analizar el proceso de cada estudiante y los diferentes momentos de la asesoría.

De acuerdo a la ANUIES (Asociación Nacional de Universidades e Instituciones de Educación Superior), se entiende que la Responsabilidad Social en las Instituciones de Educación Superior (RSIES) es:

La operación de una política de gestión académico-administrativa, definida por cada institución en el marco de su misión, sus principios y valores, para llevar a cabo

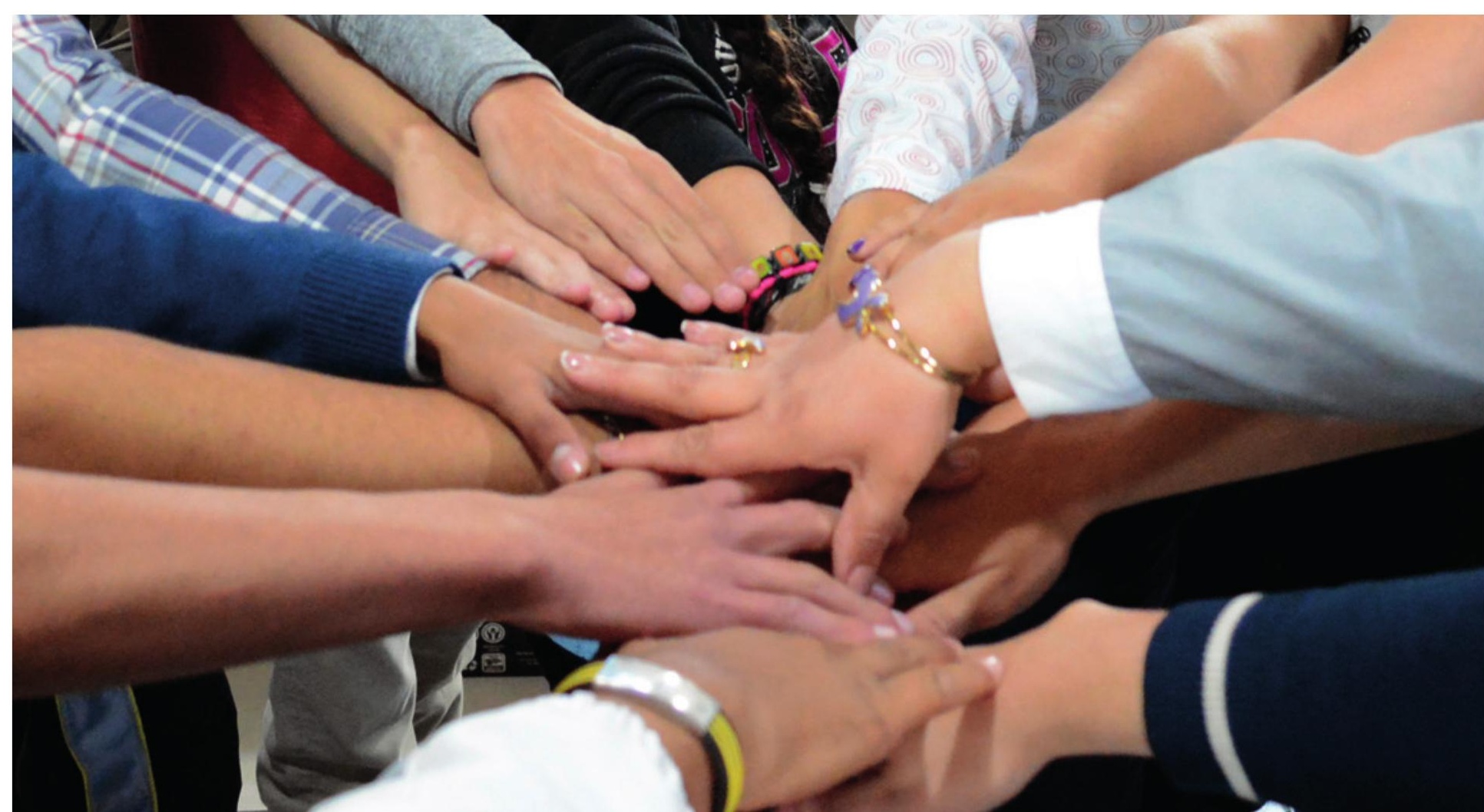


con calidad y pertinencia sus funciones, orientada al logro de resultados socialmente significativos, mediante los cuales busca contribuir al desarrollo integral y sustentable de su entorno y participar en la construcción de una sociedad más próspera, democrática y justa (ANUIES, 2017: 80).

Una vez que se reflexiona en este concepto, es alentador que se pueden realizar adaptaciones a los ambientes educativos y así contribuir a la calidad educativa, al dar la bienvenida a las necesidades actuales de nuestro entorno con una corresponsabilidad y visión periférica desde la planeación, implementación y evaluación en el arte de enseñar y transformar con atención y ética los ambientes de aprendizaje (ambientes vulnerables y diversidad de necesidades educativas), para contribuir con estrategias de integración, propiciar el bienestar socioafectivo, desarrollar metodologías activas, promover gestión institucional, fortalecer la inclusión educativa en la comunidad, formar profesionales conscientes de problemas sociales a atender, transferir conocimientos demostrativos y útiles para la vida diaria y el involucramiento de la comunidad educativa en la solución de problemas, con un beneficio pertinente y en favor de la sociedad en una "Política institucional transversal focalizada en la atención de las necesidades y expectativas sociales y de responsabilidad social" (ANUIES, 2017: 80).

\section{Conclusiones}

A partir de esta experiencia, es importante reiterar que todos los seres humanos son capaces de transformar su entorno al encontrarse en ambientes favorables de aprendizaje que los motiven en su proceso de aprendizaje por medio de la confianza y alternativas para lograr sus objetivos humanos y educativos, al reafirmar, sin duda, que un aprendizaje efectivo va de la mano de un aprendizaje afectivo; así, poder cuestionarse y encontrar inmediatamente una respuesta a la siguiente reflexión: ¿quién recuerda a un docente que dejó huella en su proceso formativo? Como actores de la educación en cualquier ámbito, la mejor obra de arte es la que se visualiza al confiar en las posibilidades de aprendizaje y dar la bienvenida a los retos que enfrentamos en el aula para contribuir en su proceso formativo, al tener siempre presente el "¿cómo?" y promover metodologías activas que, con apertura y sensibilidad al observar las necesidades del otro, se puedan implementar. Es cuando se puede decir con beneplácito que se entrelazan tres grandes conceptos: formación integral, inclusión educativa y responsabilidad social.

\section{Fuentes de consulta}

ANUIES (2017). Visión y acción 2030. Una propuesta de la ANUIES para la renovación de la educación superior en México. Diseño y concertación de políticas públicas para impulsar el cambio institucional. México: ANUIES [documento de trabajo 1.0].

Carrasco, R. (2016). La socioformación. Un enfoque para mejorar el proceso educativo universitario. [Blog]. Recuperado de: https://bit.ly/2TMOTzd.

Ceñal, C., Pérez, J. y Ortega, S. (2016). Aula inclusiva... ¿más trabajo? DOCERE, (15), 16-19. México: UAA.

Díaz, O. y Franco, F. (2010). Percepción y actitudes hacia la inclusión educativa de los docentes de Soledad, Atlántico (Colombia), 2008. Zona Próxima, (12), 12-39. Colombia: Universidad del Norte. Recuperado de: http://www. redalyc.org/articulo.oa? $\mathrm{id}=85316155002$.

González, B. (2014). Metodología socioafectiva. Una alternativa para creación de ambientes de aprendizaje trascendentes. DOCERE, (11), 27-32. México: UAA.

Hernández, J., Tobón, S. y Vázquez, J. (2014). Estudio conceptual de la docencia socioformativa. Ra Ximhai, 10(5), 89-101. El Fuerte, Sinaloa, México: Universidad Autónoma Indígena de México.

Salas, R. (2003). ¿La educación necesita realmente de la neurociencia? Estudios Pedagógicos, (29), 155.171. Chile: Universidad Austral de Chile Valdivia. Recuperado de: http://mingaonline.uach.cl/pdf/estped/n29/art11.pdf.

Significados (2019). Responsabilidad. En Signoficados.com. Consultado el 19 de septiembre de 2018, en: https://www. significados.com/responsabilidad/.

Tobón, S. (2013). Socioformación. Los retos de la educación en la sociedad de la información. Multiversidad Management, (9), 32-37. México. 